\title{
YC-1 enhances the anti-tumor activity of sorafenib through inhibition of signal transducer and activator of transcription 3 (STAT3) in hepatocellular carcinoma
}

Jian Kong ${ }^{1}$, Fandong Kong ${ }^{1}$, Jun Gao ${ }^{1}$, Qiangbo Zhang ${ }^{4}$, Shuying Dong ${ }^{1}$, Fang Gu${ }^{5}$, Shan Ke ${ }^{1}$, Bing Pan ${ }^{2}$, Qiang Shen ${ }^{2}$, Huichuan Sun ${ }^{3}$, Lemin Zheng ${ }^{2^{*}}$ and Wenbing Sun ${ }^{1 *}$

\begin{abstract}
Background: Traditional systemic chemotherapy does not provide survival benefits in patients with hepatocellular carcinoma (HCC). Molecular targeted therapy shows promise for HCC treatment, however, the duration of effectiveness for targeted therapies is finite and combination therapies offer the potential for improved effectiveness.

Methods: Sorafenib, a multikinase inhibitor, and YC-1, a soluble guanylyl cyclase (sGC) activator, were tested in HCC by proliferation assay, cell cycle analysis and western blot in vitro and orthotopic and ectopic HCC models in vivo.

Results: In vitro, combination of sorafenib and YC-1 synergistically inhibited proliferation and colony formation of HepG2, BEL-7402 and HCCLM3 cells. The combination also induced S cell cycle arrest and apoptosis, as observed by activated PARP and caspase 8 . Sorafenib and YC-1 respectively suppressed the expression of phosphorylated STAT3 (p-STAT3) (Y705) in a dose- and time-dependent manner. Combination of sorafenib and YC-1 significantly inhibited the expression of p-STAT3 (Y705) (S727), p-ERK1/2, cyclin D1 and survivin and SHP-1 activity compared with sorafenib or YC-1 used alone in all tested HCC cell lines. In vivo, sorafenib-YC-1 combination significantly suppressed the growth of HepG2 tumor xenografts with decreased cell proliferation and increased apoptosis observed by PCNA and PARP. Similar results were also confirmed in a HCCLM3 orthotopic model. There was a reduction in CD31-positive blood vessels and reduced VEGF expression, which suggested a combinational effect of sorafenib and YC-1 on angiogenesis. The reduced expression of p-STAT3, cyclin D1 and survivin was also observed with the combination of sorafenib and YC-1.
\end{abstract}

Conclusions: Our data show that sorafenib-YC-1 combination is a novel potent therapeutic agent that can target the STAT3 signaling pathway to inhibit HCC tumor growth.

Keywords: YC-1, Sorafenib, Hepatocellular carcinoma, STAT3

\footnotetext{
*Correspondence: Zhengl@bjmu.edu.cn; cyhswb@qq.com

${ }^{2}$ The Institute of Cardiovascular Sciences and Institute of Systems

Biomedicine, School of Basic Medical Sciences, Peking University Health

Science Center, Key Laboratory of Molecular Cardiovascular Sciences of

Education Ministry, and Key Laboratory of Cardiovascular Molecular Biology

and Regulatory Peptides of Health Ministry, Beijing 100191, People's Republic

of China

'Department of Hepatobiliary Surgery, Beijing Chaoyang Hospital, Capital

Medical University, Beijing 100043, People's Republic of China

Full list of author information is available at the end of the article
} 


\section{Background}

Hepatocellular carcinoma (HCC) is the sixth most common neoplasm and the third most frequent cause of cancer death [1]. Surgery, local treatment and liver transplantation may provide curative potential for patients with HCC, however, only $10-20 \%$ of patients are eligible for curative therapies [2]. Furthermore, traditional systemic chemotherapy does not provide survival benefits in patients with HCC. Molecular targeted therapy shows promise for HCC treatment. Sorafenib (Nexavar ${ }^{\circ}$ is the first and only molecular targeted therapy approved for use in HCC by the U.S. Food and Drug Administration in 2007. Sorafenib, a multiple kinase inhibitor, displays a remarkable inhibition of Raf-1 and others tyrosine kinases such as vascular endothelial growth factor receptor 2 (VEGFR2), VEGFR3, Flt-3, platelet derived growth factor (PDGF), and fibroblast growth factor recptor-1 (FGFR-1) [3]. Although sorafenib showed survival benefits in large randomized phase III studies, the response rate is actually quite low $[4,5]$. Sorafenib also causes multiple human toxicities, including use-limiting anorexia, GI bleeds and hand-foot syndrome [5]. Modulation of its actions that result in lessening of the toxicities is a desirable goal. Consequently, the duration of effectiveness for targeted therapies is finite; however, combination therapies offer the potential for improved effectiveness [6].

YC-1 [3-(5' -hydroxymethyl-2' -furyl)-1-benzylindazole] is an effective anti-platelet agent that increases cGMP levels through NO-independent activation of soluble guanylyl cyclase (sGC) and originally developed as a drug in the treatment of platelet-rich thrombosis, vasospasm or male erectile dysfunction [7]. Currently, YC-1 is also used as an inhibitor of hypoxia-inducible factor $1 \alpha$ (HIF-1 $\alpha)$ to suppress the tumor growth [8]. YC-1 exhibits anti-proliferative effects against various cancer cell lines including breast cancer [9], epithelial ovarian carcinoma [10], bladder transitional carcinoma [8], prostate cancer [11], oesophageal squamous carcinoma [12], renal carcinoma [13], lung cancer [14], leukemia [15], pancreatic cancer [16], and HCC [17-19]. YC-1 also exhibits potential prospective in treatment for $\mathrm{HCC}$. $\mathrm{YC}-1$ induced cell cycle arrest and apoptosis by activating checkpoint kinases in HCC cells $[18,19]$. YC-1 also enhanced chemo-sensitivity and eliminated the arsenic trioxide resistance in HCC [20]. Whether the combined use of YC1 and sorafenib has clinical therapeutic potential for HCC patients has not been determined.

The signal transducer and activator of transcription 3 (STAT3) has been implicated in signal transduction by different cytokines, growth factors and oncogenes. Activated STAT3 plays an important role in tumorigenesis through the upregulation of genes involved in anti-apoptosis, proliferation and angiogenesis [21]. Consequently, STAT3 has emerged as a promising target for cancer therapy, which is pharmacologically safe and effective, and blocking STAT3 activation has the potential for treatment of HCC. Recent findings showed that sorafenib inhibited tumor growth through Raf-MEK-MAPK-independent pathways and STAT3 was a major kinase-independent target of sorafenib in HCC [22], and sorafenib inhibited growth and metastasis of HCC by blocking STAT3 [23]. However, endothelial growth factor receptor (EGFR), which could activate STAT3, has been implicated both in the inherent and acquired resistance to sorafenib [24,25]. A majority of HCC patients do not respond to sorafenib, and most if not all patients who initially respond to sorafenib, subsequently become refractory and develop tumor progression after a few months of sorafenib therapy [26]. Therefore, an approach that improves therapeutic efficacy is urgently needed. Several preclinical studies have revealed the apoptosisenhancing effects of sorafenib on both hematological and solid tumor cells. YC-1 was also reported to inhibit the activity of STAT3 in HCC [17]. Whether YC-1 could be used to enhance the inhibition of activation of STAT3 of sorafenib needs to be elucidated.

In this article, we asked whether the anti-proliferative property of sorafenib and YC-1 in HCC tumors was synergic when administered in combination, and whether their concomitant use played a role through the inhibition of the STAT3 signaling pathway. We assessed the combined effects of sorafenib and YC-1 on HCC cells and xenograft models. Our results provide a rationale for combined use of sorafenib and YC-1 in HCC.

\section{Methods}

\section{Materials}

Sorafenib was kindly provided by Bayer Pharmaceuticals. YC-1 was obtained from Sigma-Aldrich (Saint Quentin Fallavier, France). Anti-PARP, anti-caspase 8 , anti- $\beta$-actin, and anti-caspase 9 were obtained from Beyotime (Jiangsu, China). Horseradish peroxidase (HRP)-labeled anti-mouse and anti-rabbit secondary antibodies were from Santa Cruz (Dallas, TX, USA). All other antibodies were purchased from Abcam (Cambridge, TX, USA).

\section{Cell lines and cell culture}

Established human HCC cell lines, HepG2 and BEL7402 were from the American Type Culture Collection (ATCC; Manassas, VA, USA). HCCLM3 was kindly provided by the Liver Cancer Institute and Zhongshan Hospital (Shanghai, China) [27]. All HCC cells were maintained in high-glucose Dulbecco's modified Eagle medium (DMEM) supplement with 10\% fetal bovine serum (FBS), $100 \mathrm{U} / \mathrm{ml}$ penicillin and $100 \mu \mathrm{g} / \mathrm{ml}$ streptomycin (Life Technologies, Cergy Pontoise, France) in a humidified atmosphere of $5 \% \mathrm{CO}_{2}$ at $37^{\circ} \mathrm{C}$. The human normal liver cell line L02 was provided by Cancer Institute \& Hospital of 
Chinese Academy of Medical Sciences, which was cultured in PRMI 1640 supplement with 10\% FBS, $100 \mathrm{U} / \mathrm{ml}$ penicillin and $100 \mu \mathrm{g} / \mathrm{ml}$ streptomycin. All cell lines were immediately expanded and frozen down such that all cell lines could be restarted every 3 months from a frozen vial of the same batch of cells. No further authentication was done. All cell lines were routinely tested to rule out mycoplasma infection.

\section{Proliferation assay}

Cell proliferation assay was measured using a Cell Counting Kit-8 (CCK-8; Dojindo Laboratories, Shanghai, China) according to the manufacturer's instructions. Briefly, cells were cultured in 96-well plates at a concentration of $3 \times 10^{3} /$ well, incubated for $24 \mathrm{~h}$, and treated with sorafenib and/or YC-1. After $72 \mathrm{~h}$ treatment, CCK8 reagent was added to each well. The absorbance was measured at $450 \mathrm{~nm}$ after $2.5 \mathrm{~h}$ incubation at $37^{\circ} \mathrm{C}$ using an automated ELISA plate reader. Any synergistic effects resulting from combination of the compounds were measured using the methods described by Chou and Talalay by using Microsoft Excel software to determine the combination index values $(\mathrm{CI}>1$ : antagonistic effect, $\mathrm{CI}=1$ : additive effect, and $\mathrm{CT}<1$ : synergistic effect) [28].

\section{Colony formation assay}

Briefly, 6-well dishes were seeded with $1 \times 10^{3}$ viable cells and allowed to grow for $24 \mathrm{~h}$. The cells were then incubated in the presence or absence of sorafenib, YC-1 and their combinations for $24 \mathrm{~h}$ in complete medium, washed with media, and allowed to grow in complete medium for 2 weeks. The colonies obtained were washed with PBS and fixed in $4 \%$ paraformaldehyde for $20 \mathrm{~min}$ at room temperature and then washed with PBS followed by staining with crystal violet. The colonies were counted and compared with untreated cells. Three different independent experiments were performed.

\section{Cell cycle analysis}

HCC cells were plated in 6-well plates at $2 \times 10^{5}$ cells/ well. Following the designated treatments, cells were harvested by trypsinization and washed with PBS and fixed in ice-cold $75 \%$ ethanol overnight at $-20^{\circ} \mathrm{C}$. Fixed cells were washed, and dissolved in RNAse and subsequently incubated at $37^{\circ} \mathrm{C}$ for $30 \mathrm{~min}$. Next, cells were stained with propidium iodide (PI) for $30 \mathrm{~min}$. The DNA content of the cells $\left(1 \times 10^{4}\right.$ cells per experimental group) was determined using a BD Accuri C6 flow cytometer (BD biosciences).

\section{Apoptosis assay}

HCC cells (HepG2, BEL-7402 or HCCLM3) were seeded in 6-well culture plates in culture medium with $2 \%$ FBS at the concentration of $2 \times 10^{5}$ cells/well. The following day, the cells were treated with sorafenib and/or YC-1 for a $48 \mathrm{~h}$ period. After treatment, cells were washed with PBS and fixed with 4\% paraformaldehyde followed by staining with Hoechst 33258, and the apoptosis cells were examined by immunofluorescence microscope, or all cells including both floating and attached cell were collected, and the apoptotic cells were detected by Annexin V-FITC Apoptosis Detection Kit (KeyGEN Biotech, Nanjing, China). The cells were stained with Annexin V-FITC and PI according to the supplier's instructions. Viable and dead cells were detected by a BD Accuri C6 flow cytometer (BD biosciences).

\section{Gene knockdown using small interfering RNA}

SMARTpool small interfering RNAs (siRNA), including control and STAT3 were synthesized by Shanghai GenePharma Co. (Shanghai, China). The procedure has been described previously [29].

\section{Western blot analysis}

Cell lysate protein content was determined using a Bicinchoninic acid (BCA) protein assay kit. Equivalent amounts of whole cell extracts, which were based on total protein content, were subjected to SDS-PAGE gel and transferred to nitrocellulose membranes. The membranes were blocked with 5\% non-fat milk for $2 \mathrm{~h}$ and then incubated with respective primary antibody overnight at $4^{\circ} \mathrm{C}$ followed by the incubation with the appropriate HRP-conjugated secondary antibody for $1.5 \mathrm{~h}$ at room temperature. Immunoreactivity was detected with SuperSignal West Pico substrate (Thermo scientific, Rockford, IL, USA).

\section{SHP-1 phosphatase activity}

The Rediplate 96 EnzChek Tyrosine Phosphatase Assay Kit (R-22067) was used for SHP-1 activity assay (Molecular Probes, Carlsbad, CA). The SHP-1 phosphatase activity was measured as described before [30].

\section{Animals}

Male BALB/c nu/nu mice (4-6 weeks of age) were obtained from Vital River Laboratories (Beijing, China) and housed under defined flora conditions in individually ventilated sterile microisolator cages. All experimental procedures using these mice were carried out in accordance with protocols approved by the Animal Care and Use Committee of Capital Medical University (Beijing, China).

\section{Orthotopic and ectopic HCC models}

In the orthotopic model, HCCLM3 cells $\left(5 \times 10^{6}\right)$ were suspended in $200 \mu \mathrm{L}$ serum-free DMEM and matrigel (1:1) and then injected subcutaneously into the upper right flank region of nude mice. When the subcutaneous 
tumor reached approximately $1 \mathrm{~cm}$ in length (approximately 4 weeks after injection), it was removed, minced into small pieces of equal volume $\left(2 \times 2 \times 2 \mathrm{~mm}^{3}\right)$, and transplanted into the livers of 20 nude mice. In the ectopic HCC model, HepG2 cells $\left(5 \times 10^{6}\right)$ were suspended in $200 \mu \mathrm{L}$ serum-free DMEM and matrigel (1:1) and then injected subcutaneously into the upper right flank region of 20 nude mice. When the tumor reached a mean size of about $100 \mathrm{~mm}^{3}$, mice were randomized into each experimental group according to tumor size, to start the treatment with a similar mean size in each group. Mice were treated with sorafenib by oral route $(30 \mathrm{mg} / \mathrm{kg} /$ day $)$, or intraperitoneal YC-1 (10 mg/kg/day), or combination of sorafenib and $\mathrm{YC}-1$, or DMSO and polyoxyethylenated castor oil as control every day for up to the 24th day. Tumor size was measured with a caliper rule every 3 days. The tumor volume was calculated as follows: TV $\left(\mathrm{mm}^{3}\right)=$ $\left(\mathrm{L} \times \mathrm{W}^{2}\right) / 2$, where $\mathrm{L}$ was the longest and $\mathrm{W}$ the shortest radius of the tumor in millimeters. At the end of the experiments, mice were euthanized, blood samples were collected via cardiac puncture, and tumor tissues were removed for fixation in the $4 \%$ paraformaldehyde for histologic examination and immunohistochemical staining.

\section{Immunocytochemistry}

Tissues were fixed in $4 \%$ paraformaldehyde and subsequently embedded in paraffin. Paraffin-embedded tissue sections were cut into standard $6 \mu \mathrm{m}$ sections, deparaffinized in xylene and rehydrated through graded alcohol solutions. Antigen retrieval was performed $10 \mathrm{~min}$ at $92^{\circ} \mathrm{C}$ in EDTA (10 mmol/l, pH 8.0) in a water bath. Endogenous peroxidases were inactivated by immersing the sections in $0.3 \%$ hydrogen peroxide for $12 \mathrm{~min}$. The sections were blocked with $5 \%$ goat serum for $60 \mathrm{~min}$ at $37^{\circ} \mathrm{C}$. The slides were incubated with primary antibodies for overnight at $4^{\circ} \mathrm{C}$. Next, the slides were treated with appropriate HRPconjugated secondary antibody for $40 \mathrm{~min}$ at $37^{\circ} \mathrm{C}$ and then developed with 3,3'-diaminobenzidine. Finally, the slides were counterstained with hematoxylin and mounted. The slides were examined with Nikon Eclipse Ti microscope under a $200 \times$ objective.

\section{Statistical analysis}

All values are expressed as the mean \pm SEM. The data were analyzed using Student's $t$ test or the ANOVA test. A P value of $<0.05$ was considered statistically significant. GraphPad Prism (GraphPad Software Inc., San Diego, California, USA) was used for these analyses.

\section{Results}

\section{Combination of sorafenib and YC-1 inhibited HCC cell proliferation}

To determine the growth inhibition effect of combination of sorafenib and YC-1, HCC cell lines HepG2,
BEL-7402 and HCCLM3 were incubated for $72 \mathrm{~h}$ with sorafenib and/or YC-1. Chemical structures of sorafenib and $\mathrm{YC}-1$ were shown in Additional file 1: Figure S1. Sorafenib or $\mathrm{YC}-1$ alone inhibited $\mathrm{HCC}$ cell proliferation in a dose-dependent manner (Figure 1A). Moreover, combination of sorafenib and $\mathrm{YC}-1$ significantly suppressed proliferation of HCC cells in a dose-dependent manner (Figure 1B). To determine the long-term combination effect of sorafenib-YC-1 treatment, cells were incubated with sorafenib and/or YC-1 for $24 \mathrm{~h}$, washed with media, and allowed to grow in complete medium for 2 weeks. There was lower number of colonies in the combination compared with other treatments (Figure 1C). In addition, at the $\mathrm{ED}_{50}$ doses for both sorafenib and $\mathrm{YC}-1$, we found that $\mathrm{CI}=0.93$ in HepG2, 0.95 in BEL-7402 and 0.72 in HCCLM3 respectively, suggesting that sorafenib and YC-1 synergistically inhibited proliferation of HCC cells (Figure 1D). These data suggested that combination of sorafenib and $\mathrm{YC}-1$ treatment synergistically suppresses proliferation of $\mathrm{HCC}$ cells in vitro. Otherwise, we determined the effect of the combination on proliferation of the human normal liver cell line L02. Sorafenib (5 $\mu \mathrm{M})$ significantly inhibited $(66.9 \%$ decrease) the proliferation of L02 cells (Additional file 2: Figure S2). Although YC-1 $(20 \mu \mathrm{M})$ significantly suppressed the proliferation of L02 cells, only $12.1 \%$ decrease was observed (Additional file 2: Figure S2). YC-1 could not increase the toxicity of sorafenib to L02 cells (Additional file 2: Figure S2).

\section{The sorafenib-YC-1 combination induced cell cycle arrest and apoptosis}

Given their effects on cell proliferation, we then carried out cell-cycle and apoptosis analysis to further characterize sorafenib-YC-1 combination effects. HepG2 cell populations in the G0-G1 and S phases were $30.72 \%$ and $63.93 \%$ in the combination of sorafenib and $\mathrm{YC}-1$ group, while $77.42 \%$ and $17.63 \%$ in sorafenib $(5 \mu \mathrm{M})$ group, and $45.69 \%$ and $45.12 \%$ in $\mathrm{YC}-1$ group (Figure $2 \mathrm{~A}$ ). These results suggested that sorafenib-YC-1 combination significantly induced $S$ phase arrest compared with sorafenib or YC-1 used alone in HepG2 cells. At 48 h, sorafenibYC-1 combination significantly induced apoptosis in HepG2 cells (Figure 2B). Hoechst 33258 staining further confirmed results above (Figure $2 \mathrm{C}$ ). Similar results were also found in BEL-7402 and HCCLM3 cells (data of apoptosis assay not shown) (Additional file 3: Figure S3). Furthermore, we determined the mechanism of cell death. Increased activation of PARP was observed within $24 \mathrm{~h}$ in all HCC cells treated with sorafenib-YC-1 combination (Figure 2D). The apoptotic signaling pathways are generally divided into two types: the extrinsic or death receptor pathway, which activates caspase 8 , and the intrinsic or mitochondrial pathway, which triggers the activation of 

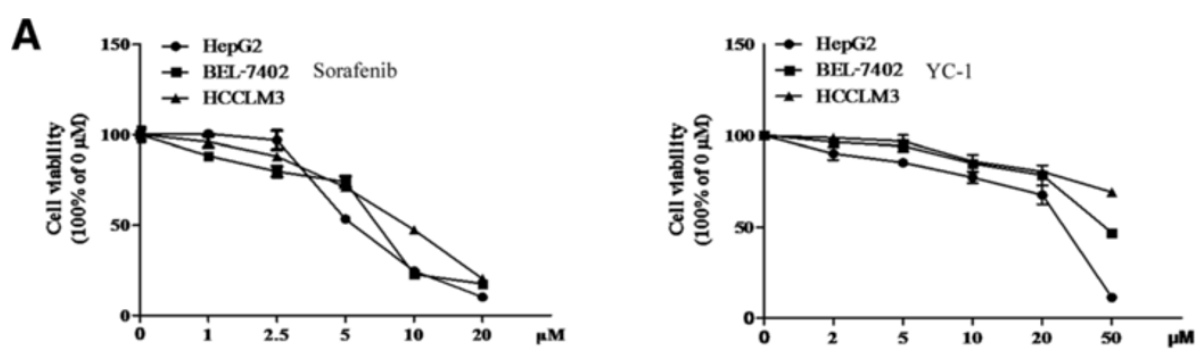

B
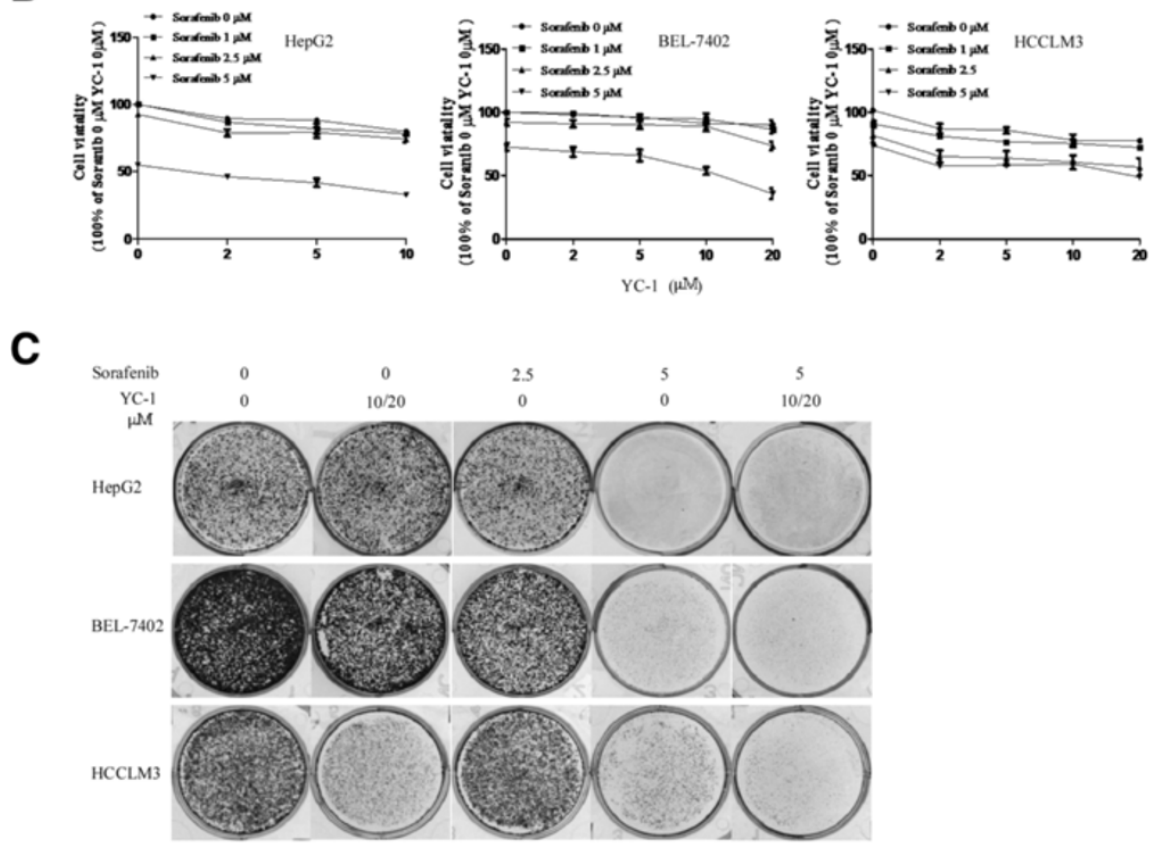

D
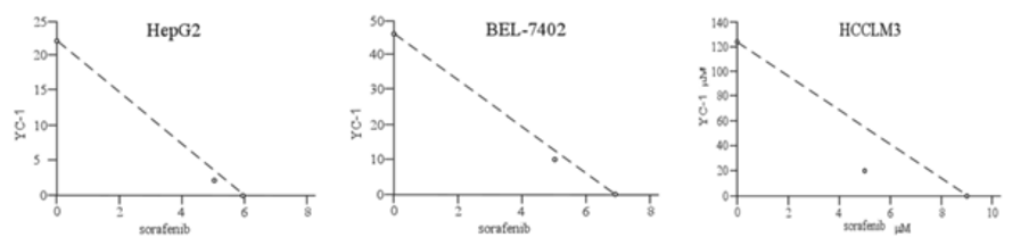

Figure 1 Sorafenib-YC-1 combination synergistically inhibited proliferation of HCC cells. A, HepG2, BEL-7402 and HCCLM3 cells were incubated with increasing doses of sorafenib $(0-20 \mu \mathrm{mol} / \mathrm{L})$ or YC-1 (0-50 $\mu \mathrm{mol} / \mathrm{L})$ for up to $72 \mathrm{~h}$. Data were presented as percentages of cell proliferation as

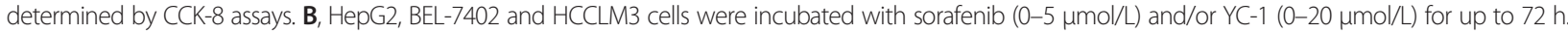
Data were presented as percentages of cell proliferation as determined by CCK-8 assays. C, HepG2, BEL-7402 and HCCLM3 cells were treated sorafenib $(2.5 \mu \mathrm{mol} / \mathrm{L}$ or $5 \mu \mathrm{mol} / \mathrm{L})$ or $\mathrm{YC}-1(10 \mu \mathrm{mol} / \mathrm{L}$ or $20 \mu \mathrm{mol} / \mathrm{L})$ or in combination. The colonies were assessed. $\mathbf{D}$, Synergisms of proliferation inhibition of different cell lines were analyzed by isobologram analysis. Error bars represent the SEM of data obtained in at least three independent experiments.

caspase 9. Increased activation of caspase 8 not caspase 9 was observed in HCC cells treated with sorafenib-YC-1 combination (Figure 2D). These data suggested that combination of sorafenib and $\mathrm{YC}-1$ is a potent inducer of apoptosis of HCC cells involved in activation of the extrinsic apoptosis pathway.

\section{The sorafenib-YC-1 combination suppressed STAT3 phosphorylation}

We investigated the levels of phosphorylated STAT3 and p44/42 MAPK (ERK1/2) in HepG2, BEL-7402 and
HCCLM3 cells after sorafenib and/or YC-1 treatment. Phosphorylated STAT3 (p-STAT3) (Y705) and phosphorylated ERK1/2 (p-ERK1/2) were reduced at both an early time point $(4 \mathrm{~h})$ and a late time point $(24 \mathrm{~h})$ following sorafenib treatment in a dose-dependent manner (Additional file 4: Figure S4). Furthermore, p-STAT3 at Y705 was also decreased at $4 \mathrm{~h}$ and $24 \mathrm{~h}$ following YC-1 treatment in a dose-dependent manner (Additional file 4: Figure S4). However, p-ERK1/2 was not significantly changed at early time point following $\mathrm{YC}-1$ treatment. And at a late time point, p-ERK1/2 was not 


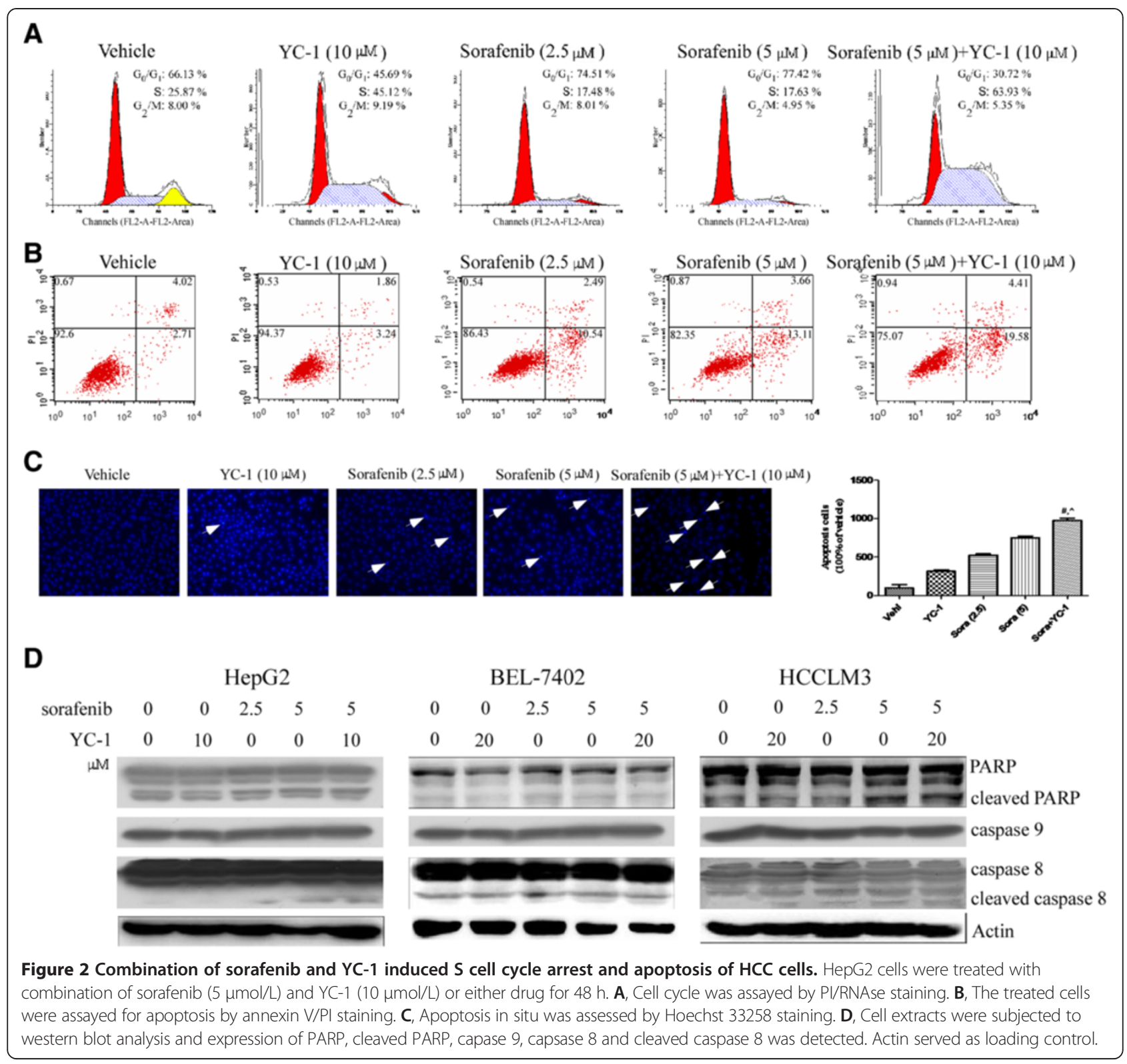

changed in HepG2 cells and significantly changed in BEL-7402 only at the concentration of $50 \mu \mathrm{M}$ YC-1 (Additional file 4: Figure S4). Moreover, p-ERK1/2 was reduced in a dose-dependent manner at a late time point following YC-1 treatment in HCCLM3 cells (Additional file 4: Figure S4). Interestingly, combination of sorafenib and YC-1 significantly inhibited the p-STAT3 (Y705) (S727) and p-ERK1/2 compared with sorafenib or YC-1 used alone at $24 \mathrm{~h}$ (Figure $3 \mathrm{~A}$ and Additional file 5: Figure S5). Sorafenib-YC-1 combination also suppressed the expression of cyclin D1 and survivin in all tested HCC cell lines (Figure 3A).

Furthermore, both YC-1 and sorafenib increased SHP1 activity in comparison with control cells, and the combination of sorafenib and YC-1 significantly increased
SHP-1 activity compared with sorafenib or YC-1 alone in HepG2 and BEL-7402 cells (Figure 3B).

Furthermore, STAT3 siRNA was used to delete STAT3, and the expression of p-STAT3 (Y705) and STAT3 was significantly decreased in HepG2 and BEL7402 cells (Additional file 6: Figure S6A). Deletion of STAT3 could not rescue HepG2 and BEL-7402 cells from apoptosis. Inversely, silencing STAT3 improved the sensitivity of HepG2 and BEL-7402 cells to the combination of sorafenib and YC-1 (Additional file 6: Figure S6B).

The sorafenib-YC-1 combination inhibited tumor growth in vivo To evaluate the role of sorafenib-YC-1 combination on tumor growth in vivo, we examined their effects in a HepG2 ectopic HCC model and HCCLM3 orthotopic 


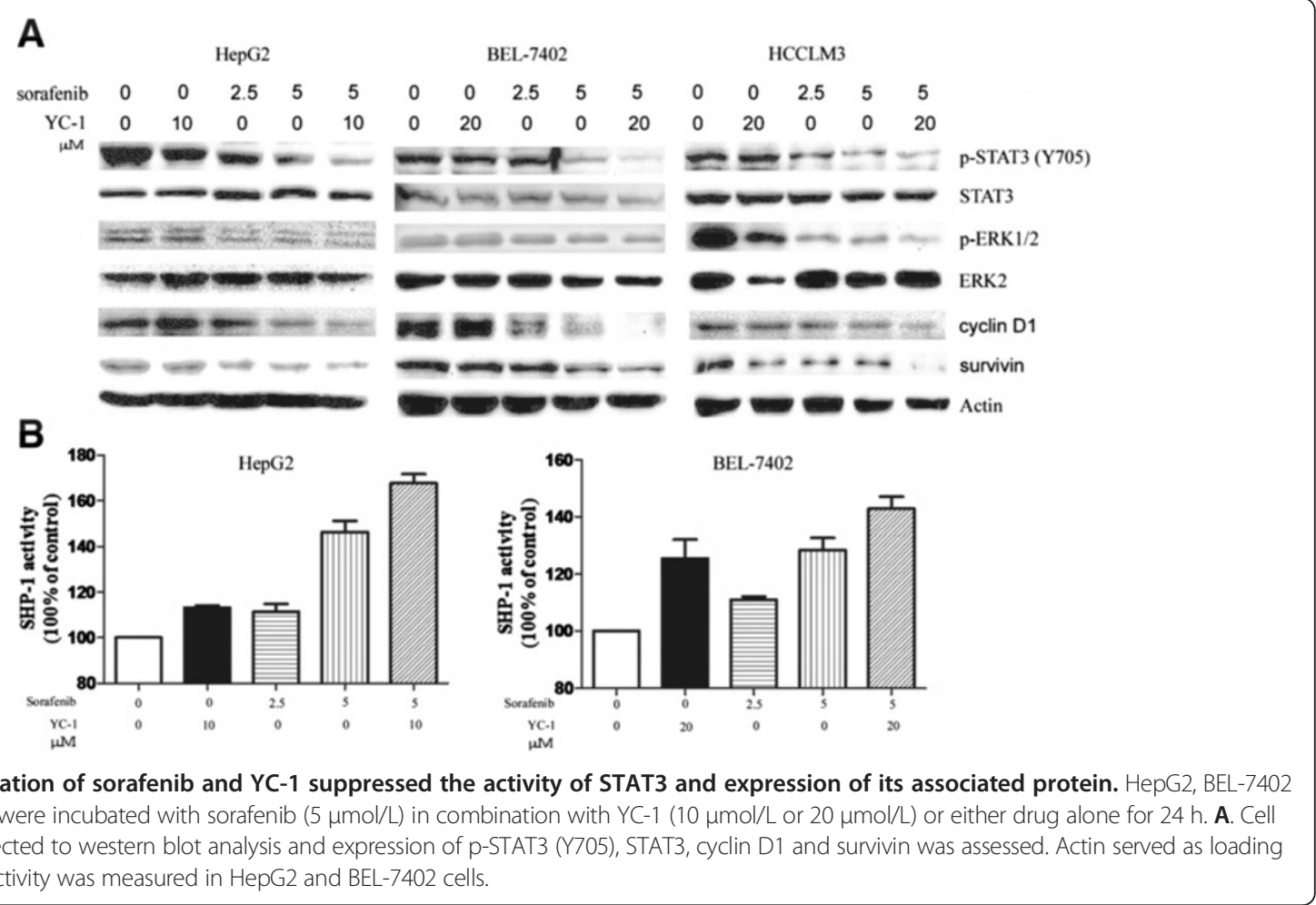

model. Sorafenib or YC-1 inhibited the growth of the HepG2 xenografts, and their combination exhibited an enhanced effect (Figure 4A). Similar results were observed in HCCLM3 orthotopic model (Additional file 7: Figure S7). The excised tumors from vehicle mice, mice treated with either sorafenib or YC-1 alone and their combination weighed approximately $2000 \mathrm{mg}, 1500 \mathrm{mg}, 1200 \mathrm{mg}$ and $800 \mathrm{mg}$ respectively, which suggested significant effect when the mice were treated with the combination of drugs (Figure $4 \mathrm{~B}$ ). When tumors were treated with the combination of sorafenib and $\mathrm{YC}-1$, significant decreases of cell proliferation and increases of apoptosis were observed by PCNA and PARP (Figure 4C and Additional file 8: Figure S8). There were no apparent changes in liver, heart, kidney, levels of serum glutamic-pyruvic transaminase (GOT) and glutamic oxalacetic transaminase (GST) and body weight in the mice (Additional file 9: Figure S9A, $\mathrm{B}$ and $\mathrm{D})$. But the mice treated with sorafenib alone showed significant decreased spleen volume $(0.0702 \pm 0.003247 \mathrm{~g})$ compared with control group $(0.1050 \pm 0.007266 \mathrm{~g}), \mathrm{YC}-1$ group $(0.0992 \pm 0.000860 \mathrm{~g})$ or sorafenib $+\mathrm{YC}-1$ group $(0.08660 \pm 0.003530 \mathrm{~g})$ (Additional file 9: Figure S9C). These data implied that the sorafenib-YC-1 may be a potential therapeutic combination for treating $\mathrm{HCC}$, and relatively nontoxic to the animals.

Sorafenib and YC-1 combination inhibited the STAT3 signaling associated proteins and tumor angiogenesis in vivo

Furthermore, treatment with the sorafenib and YC-1 combination led to a significant reduction in p-STAT3, cyclin D1, and survivin compared with sorafenib or YC-1 used alone (Figure 4D and Additional file 8: Figure S8). Endothelial-specific antigen CD31 and VEGF levels were also significantly lower in sorafenib-YC-1 combinationtreated tumor xenografts (Figure 4E and Additional file 8: Figure S8).

\section{Discussion}

HCC typically arises on the basis of cirrhosis and responds poorly to conventional cytotoxic chemotherapy [31]. This has led to a search for novel approaches for molecular targeted therapy. The RAF/MEK/ERK cascade is one of the principal RAS-regulated pathways. Raf expression has been reported to be increased in human HCC, and sorafenib was synthesized to molecularly target RAF in this vital pathway and has been shown to have anti-tumor activity against renal cell cancer and HCC. However, recent researches have shown that STAT3 is a vital target of sorafenib and its effect is not correlated to the repression to Raf-1 kinase [22,23]. In addition, sorafenib causes multiple toxicities and a large percent of patients need to be treated with a reduced dose or stop taking the drug for this reason [32]. So we wanted to search for new agents that could be combined with sorafenib to reduce the dose dosage of sorafenib and enhance its HCC growth inhibition through enhanced suppressing STAT3 activity. In the present study, we showed not only the combined anti-tumor effects of low-dose sorafenib and YC-1 therapy against $\mathrm{HCC}$ both 


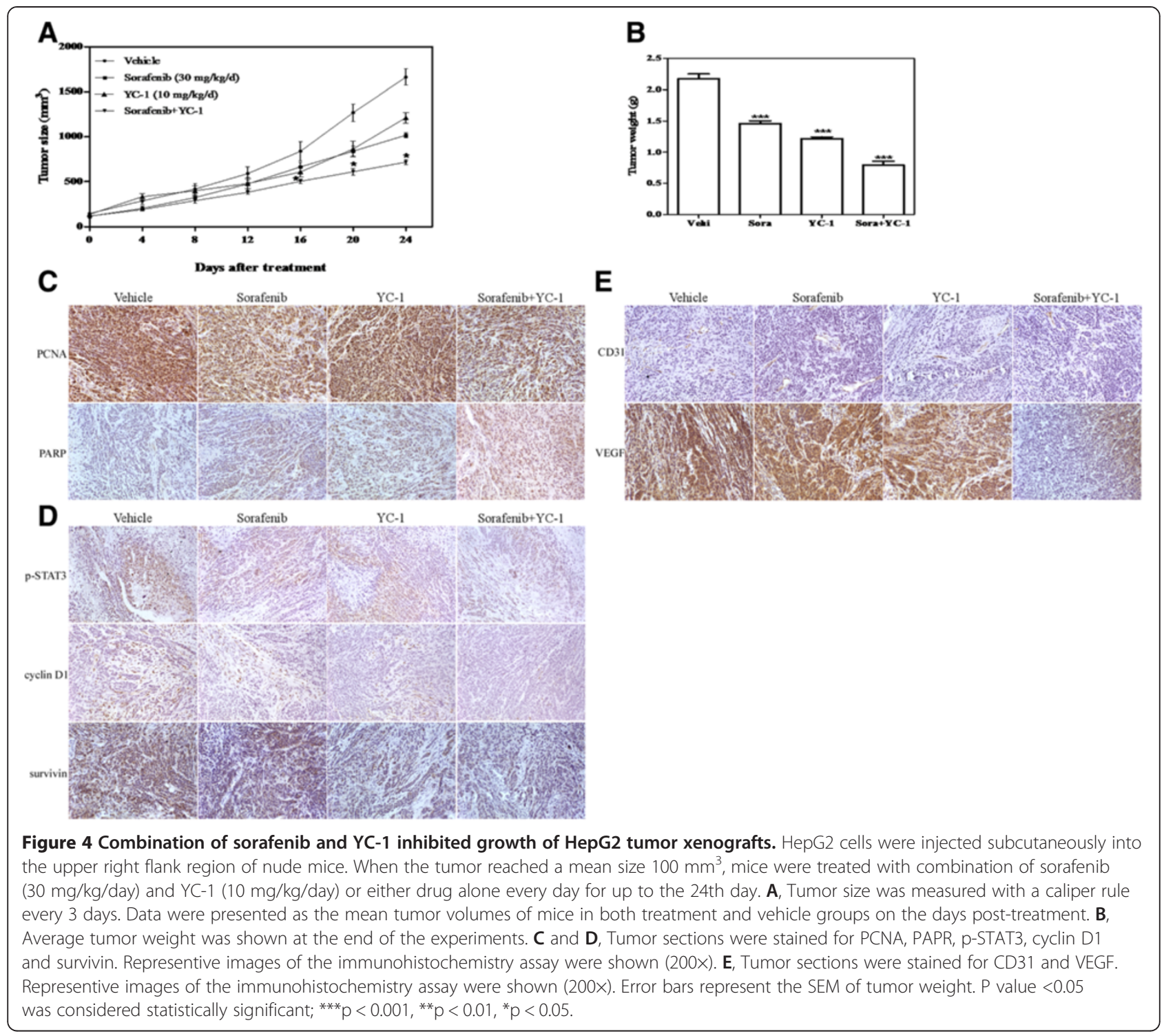

in vitro and in vivo, but also the novel mechanism through which YC-1 sensitized HCC cells to sorafenib. Our results indicated that the sorafenib-YC-1 combination possesses potential as a promising therapeutic agent against HCC.

Sorafenib is the first and only molecular targeted therapy approved for HCC and could inhibit proliferation of HCC cells by inducing apoptosis [33]. However, the poor response rate renders the drug less than satisfactory. In this study, YC-1 was used to enhance its inhibition. We found that sorafenib-YC-1 combination synergistically inhibited proliferation of HCC cells. Apoptosis and cell cycle analysis was further used to elucidate the mechanism involved in the synergistical effect of sorafenib and $\mathrm{YC}-1$. Combination of sorafenib and YC-1 induced more apoptosis (23.99\%) compared with sorafenib (16.77\%) or YC-1 (5.1\%) alone. Apoptosis rate was limited, which may not explain synergistical effect of sorafenib and $\mathrm{YC}$ 1. Our data demonstrated that YC-1 significantly induced $\mathrm{S}$ cell cycle arrest compared with the vehicle, and sorafenib increased the $\mathrm{S}$ cell cycle arrest of $\mathrm{YC}-1$ in all HCC cells. Interestingly, Eun-Jin Yeo et al. (2006) suggested that YC-1 induced S cell cycle arrest and apoptosis by activating checkpoint kinases in Hep3B cells [18]. However, Wang SW et al. (2005) showed that YC-1 exhibited an anti-proliferative effect and arrested the cell cycle in G0-G1 in human HCC cells [19]. The reasons may be contributed to the different doses of $\mathrm{YC}-1$ in the studies. Our results were consistent with Eun-jin Yeo el al. and further suggested that sorafenib could enhance the $\mathrm{S}$ cell cycle arrest of $\mathrm{YC}-1$. Our data indicated that apoptosis and $\mathrm{S}$ cell cycle induced by sorafenib-YC-1 combination may be the mechanism of their synergistical inhibition of proliferation in human HCC cells. 
STAT3 has a critical role in liver inflammation and tumor progression because it can be triggered by cytokines and growth factors such EGFR, FGFR and PDGF through tyrosine phosphorylation [34]. Many STAT3related genes such as cyclin D1 and survivin also play roles in cell proliferation and survival signaling [35]. Constitutive activation of STAT3 is observed in $72.4 \%$ of human HCC and in a wide variety of other cancer types not in normal cells [36], which represents an attractive molecular target. In HCC, STAT3 frequently correlates with the constitutive upregulation of Ras implicated in HCC progression [37]. The anti-tumor activity of sorafenib does not necessarily correlate with the inhibition of ERK phosphorylation in HCC cells [25]. Previous studies also reported that STAT3 was the key factor in the mechanism of sorafenib resistance, and STAT3 rather than Raf- 1 was critical in the anti-cancer effect of sorafenib [30]. Our studies showed that YC-1 or sorafenib alone suppressed the activity of STAT3 in a time- and dose-dependent manner. Furthermore, combination of sorafenib and YC-1 significantly inhibited the p-STAT3 (Y705) (S727) compared with sorafenib or YC-1 alone. Meantime, YC-1 did not inhibit the activity of p-ERK1/2 or only performed at a high dose $(50 \mu \mathrm{M})$, which may be not significant in the clinic. However, combination of sorafenib and YC-1 significantly suppressed p-ERK1/2 in all tested HCC cell lines, whose mechanism needed to be determined in the future. Sorafenib suppresses the activity of STAT3 through enhancing SHP-1 activity [30]. Our results showed that combination of sorafenib and $\mathrm{YC}-1$ may suppress the expression of p-STAT3 through inhibiting the of SHP-1 activity. And sorafenbYC-1 combination also significantly suppressed the expression of cyclin D1 and survivin. These results indicated that YC-1 may be used to enhance the inhibition of STAT3 activity of sorafenib, and further suppress the HCC growth (Figure 5).

We examined the in vivo effect of sorafenib-YC-1 combination in HCC xenografts in nude mice. We demonstrated that combination of sorafenib $(30 \mathrm{mg} / \mathrm{kg} / \mathrm{d})$ and $\mathrm{YC}-1(10 \mathrm{mg} / \mathrm{kg} / \mathrm{d})$ could significantly suppress the growth of HCC tumor when compared with either drug alone. Of note, apparent toxicity was not observed in heart, lung, liver and kidney. But shrank spleen were found in the sorafenib group and the mechanism involved should be further elucidated to assure the safe use. Also, the treatment allowed the mice to maintain normal weight gain and levels of serum GOP and GPT. Our studies showed that combination of sorafenib and YC-1 could be safe to be used in vivo, which provided the preliminary basis for potential use for HCC patients in the future. Furthermore, combination of sorafenib and $\mathrm{YC}-1$ also suppressed the expression of VEGF and microvessel density (CD31) in HCC tumor compared

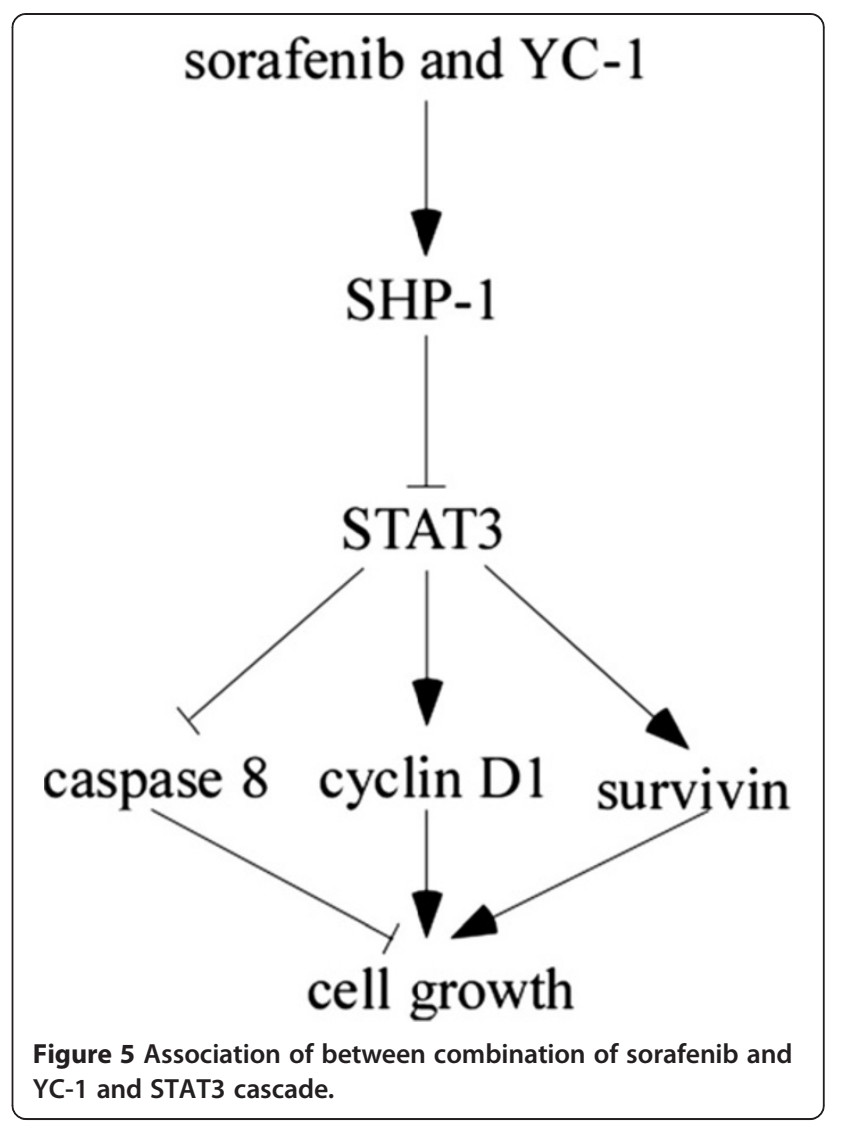

with either drug alone. VEGF is the most potent angiogenic factor and plays a key role in tumor associated angiogenesis and hyper-permeability [38]. Studies have shown that VEGF is frequently expressed in HCC [29]. Our data suggested that sorafenib-YC-1 combination to inhibit VEGF and CD31 expression may be another molecular mechanism to prevent HCC growth. Moreover, Liang et al. recently showed that anti-angiogenic activity of sorafenib could be responsible for the activation of resistance mechanisms, sustained sorafenib treatment led to increased intratuour hypoxia and induction of HIF- $1 \alpha$ expression that mediated cell survival, and the use of HIF-1 $\alpha$ inhibitors (EF24) abolished drug resistance [39]. $\mathrm{YC}-1$ is also a HIF- $1 \alpha$ inhibitor, so $\mathrm{YC}-1$ may play a assisting role in anti-tumor of sorafenib through the mechanism above, which confirms us that sorafenib-YC1 combination could be potential for use for HCC patients in near future.

\section{Conclusions}

Our results revealed that $\mathrm{YC}-1$ has a synergistic effect with sorafenib on HCC through inhibition of STAT3 activity and that the STAT3 signaling pathway may be a suitable target for the development of anti-HCC targeted agents. 


\section{Additional files}

\section{Additional 1: Figure S1. Chemical structures of sorafenib and YC-1} were shown.

Additional 2: Figure S2. The effect of sorafenib and $Y C-1$ on the proliferation of L02 cells. L02 cells were incubated with sorafenib $(0-5 \mu \mathrm{mol} / \mathrm{L})$ and/or YC-1 (0-20 $\mathrm{mmol} / \mathrm{L})$ for up to $72 \mathrm{~h}$. Data were presented as percentages of cell proliferation as determined by CCK-8 assays.

Additional 3: Figure S3. Combination of sorafenib and $Y C-1$ induced $S$ cell cycle arrest and apoptosis of HCC cells. BEL-7402 and HCCLM3 cells were treated with combination of sorafenib $(5 \mu \mathrm{mol} / \mathrm{L})$ and $Y C-1$ $(20 \mu \mathrm{mol} / \mathrm{L})$ or either drug for 48 h. A, Cell cycle was assayed by PI/RNAse staining in BEL-7402 and HCCLM3 cells. B, The treated cells were assayed for apoptosis by annexin V/PI staining in BEL-7402 cells. C, Apoptosis in situ was assessed by Hoechst 33258 staining in BEL-7402 and HCCLM3 cells.

Additional 4: Figure S4. Sorafenib and YC-1 affected STAT3 and ERK1/2 pathways. HepG2, BEL-7402 and HCCLM3 cells were treated with increasing doses of sorafenib $(0-20 \mu \mathrm{mol} / \mathrm{L})$ or $Y C-1(0-50 \mu \mathrm{mol} / \mathrm{L})$ for up to $4 \mathrm{~h}$ or $24 \mathrm{~h}$. Cell extracts were subjected to western blot analysis and expression of p-ERK1/2, ERK2, p-STAT3 (Y705), STAT3 was detected. Actin served as loading control.

Additional 5: Figure S5. Combination of sorafenib and $Y C-1$ suppressed the activity of STAT3 (S727). HepG2, BEL-7402 and HCCLM3 cells were incubated with sorafenib $(5 \mu \mathrm{mol} / \mathrm{L})$ in combination with $\mathrm{YC}-1$ $(10 \mu \mathrm{mol} / \mathrm{L}$ or $20 \mu \mathrm{mol} / \mathrm{L})$ or either drug alone for $24 \mathrm{~h}$. Cell extracts were subjected to western blot analysis and expression of p-STAT3 (S727) and STAT3 was assessed. Actin served as loading control.

Additional 6: Figure S6. Down-regulation of STAT3 sensitized HCC cells to combination of sorafenib and YC-1. A, siRNA was used to silence the expression of STAT3 in HepG2 and BEL-7402 cells. The expression of p-STAT3 (Y705) and STAT3 was assessed by western blot. Actin served as loading control. B, After HepG2 and BEL-7402 cells were transferred with STAT3 siRNA, sorafenib and YC-1 were added to treat the HepG2 and BEL-7402 cells. Apoptosis cells were analyzed by annexin V/PI staining

Additional 7: Figure S7. Combination of sorafenib and YC-1 inhibited growth of orthotopic HCCLM3 tumor. HCCLM3 cells were injected subcutaneously into the upper right flank region of nude mice. When the subcutaneous tumor reached approximately $1 \mathrm{~cm}$ in length, it was minced into small pieces of equal volume, and transplanted into the livers of 20 nude mice. When the tumor reached a mean size of about $100 \mathrm{~mm}^{3}$, mice were treated with combination of sorafenib $(30 \mathrm{mg} / \mathrm{kg}$ / day) and YC-1 (10 mg/kg/day) or either drug alone every day for up to the 24th day. Representative image of tumor volume was displayed.

Additional 8: Figure S8. The expression of PCNA, PARP, p-STAT3 (Y705), cyclin D1, survivin, CD31 and VEGF was quantified by Image-Pro Plus.

Additional 9: Figure S9. Toxicity of sorafenib and/or YC-1 on nude mice bearing with tumor. HepG2 cells were injected subcutaneously into the upper right flank region of nude mice. When the tumor reached mean size of about $100 \mathrm{~mm}^{3}$, mice were treated with combination of sorafenib (30 mg/kg/day) and YC-1 (10 mg/kg/day) or either drug alone every day for up to the 24th day. A, Mice weight was measured with a scale every 3 days. B, The levels of serum GOP and GPT were shown at the end of the experiments. Error bars represent the SEM of concentration of GOP and GPT. C, Spleen weight was measured at the end of the experiments. D, Heart, lung, liver and kidney sections were stained with haematoxylin and eosin (HE). Representive images were shown (200x). ns, no significance.

\section{Competing interests}

The authors declare that they have no competing interests.

\section{Authors' contributions}

JK and FDK carried out the experiments and drafted the manuscript. JG, QBZ, FG, BP and SYD participated in the sequence alignment. LMZ and WBS conceived the study and coordination and helped to draft the manuscript. SK, QS and HCS participated in the design of the study. All authors read and approved the final manuscript.

\section{Acknowledgements}

This work was supported by Grants 320675007131 and 32067501207 from the Dr. Jieping Wu Medical Foundation, the Program for Medical Key Discipline of Shijingshan District, Beijing, 2010CB912504 and 2011CB503900 from "973" National S\&T Major Project, 81170101, 81370235 and 30821001 from the National Natural Science Foundation of China, and 7122106 from the Natural Science Foundation of Beijing, China. The authors thank Li Chen for her advice on immunocytochemistry.

\section{Author details}

'Department of Hepatobiliary Surgery, Beijing Chaoyang Hospital, Capital Medical University, Beijing 100043, People's Republic of China. ${ }^{2}$ The Institute of Cardiovascular Sciences and Institute of Systems Biomedicine, School of Basic Medical Sciences, Peking University Health Science Center, Key Laboratory of Molecular Cardiovascular Sciences of Education Ministry, and Key Laboratory of Cardiovascular Molecular Biology and Regulatory Peptides of Health Ministry, Beijing 100191, People's Republic of China. ${ }^{3}$ Liver Cancer Institute, Zhongshan Hospital, Fudan University, Shanghai 200032, People's Republic of China. ${ }^{4}$ Department of General Surgery, Qilu Hospital, Shandong University, Jinan 250012, People's Republic of China. ${ }^{5}$ Department of Obstetrics and Gynecology, Chinese PLA General Hospital, Beijing 100853, People's Republic of China.

Received: 3 August 2013 Accepted: 6 January 2014

Published: 13 January 2014

\section{References}

1. Forner A, Llovet JM, Bruix J: Hepatocellular carcinoma. Lancet 2012, 379:1245-1255

2. Verslype C, Van Cutsem E, Dicato M, Arber N, Berlin JD, Cunningham D, De Gramont A, Diaz-Rubio E, Ducreux M, Gruenberger T, et al: The management of hepatocellular carcinoma. Current expert opinion and recommendations derived from the 10th world congress on gastrointestinal cancer, Barcelona, 2008. Ann Oncol 2009, 20(Suppl 7):vii1-vii6.

3. Liu L, Cao Y, Chen C, Zhang X, McNabola A, Wilkie D, Wilhelm S, Lynch M, Carter C: Sorafenib blocks the RAF/MEK/ERK pathway, inhibits tumor angiogenesis, and induces tumor cell apoptosis in hepatocellular carcinoma model PLC/PRF/5. Cancer Res 2006, 66:11851-11858.

4. Cheng AL, Kang YK, Chen Z, Tsao CJ, Qin S, Kim JS, Luo R, Feng J, Ye S, Yang TS, et al: Efficacy and safety of sorafenib in patients in the Asia-Pacific region with advanced hepatocellular carcinoma: a phase III randomised, double-blind, placebo-controlled trial. Lancet Oncol 2009, 10:25-34

5. Llovet JM, Ricci S, Mazzaferro V, Hilgard P, Gane E, Blanc JF, de Oliveira AC, Santoro A, Raoul JL, Forner A, et al: Sorafenib in advanced hepatocellular carcinoma. N Engl J Med 2008, 359:378-390

6. LoRusso PM, Canetta R, Wagner JA, Balogh EP, Nass SJ, Boerner SA, Hohneker J: Accelerating cancer therapy development: the importance of combination strategies and collaboration. Summary of an institute of medicine workshop. Clin Cancer Res 2012, 18:6101-6109.

7. Chun YS, Yeo EJ, Park JW: Versatile pharmacological actions of YC-1: anti-platelet to anticancer. Cancer Lett 2004, 207:1-7.

8. Li Y, Zhao X, Tang H, Zhong Z, Zhang L, Xu R, Li S, Wang Y: Effects of YCon hypoxia-inducible factor 1 alpha in hypoxic human bladder transitional carcinoma cell line T24 cells. Urol Int 2012, 88:95-101.

9. Fallahian F, Karami-Tehrani F, Salami S, Aghaei M: Cyclic GMP induced apoptosis via protein kinase $\mathrm{G}$ in oestrogen receptor-positive and -negative breast cancer cell lines. FEBS J 2011, 278:3360-3369.

10. Lee CS, Kwak SW, Kim YJ, Lee SA, Park ES, Myung SC, Kim W, Lee MS, Lee JJ: Guanylate cyclase activator YC-1 potentiates apoptotic effect of licochalcone A on human epithelial ovarian carcinoma cells via activation of death receptor and mitochondrial pathways. Eur J Pharmacol 2012, 683:54-62.

11. Huang YT, Pan SL, Guh JH, Chang YL, Lee FY, Kuo SC, Teng CM: YC-1 suppresses constitutive nuclear factor-kappaB activation and induces apoptosis in human prostate cancer cells. Mol Cancer Ther 2005, 4:1628-1635.

12. Feng $Y$, Zhu H, Ling T, Hao B, Zhang G, Shi R: Effects of $Y C-1$ targeting hypoxia-inducible factor 1 alpha in oesophageal squamous carcinoma cell line Eca109 cells. Cell Biol Int 2011, 35:491-497.

13. Wu SY, Pan SL, Chen TH, Liao CH, Huang DY, Guh JH, Chang YL, Kuo SC, Lee FY, Teng CM: YC-1 induces apoptosis of human renal carcinoma A498 cells in vitro and in vivo through activation of the JNK pathway. $\mathrm{Br}$ J Pharmacol 2008, 155:505-513. 
14. Chen CJ, Hsu MH, Huang LJ, Yamori T, Chung JG, Lee FY, Teng CM, Kuo SC: Anticancer mechanisms of YC-1 in human lung cancer cell line, $\mathrm{NCl}-\mathrm{H} 226$. Biochem Pharmacol 2008, 75:360-368.

15. Chung JG, Yang JS, Huang LJ, Lee FY, Teng CM, Tsai SC, Lin KL, Wang SF, Kuo SC: Proteomic approach to studying the cytotoxicity of YC-1 on U937 leukemia cells and antileukemia activity in orthotopic model of leukemia mice. Proteomics 2007, 7:3305-3317.

16. Zhao Q, Du J, Gu H, Teng X, Zhang Q, Qin H, Liu N: Effects of YC-1 on hypoxia-inducible factor 1-driven transcription activity, cell proliferative vitality, and apoptosis in hypoxic human pancreatic cancer cells. Pancreas 2007, 34:242-247.

17. Lau CK, Yang ZF, Lam SP, Lam CT, Ngai P, Tam KH, Poon RT, Fan ST: Inhibition of Stat3 activity by YC-1 enhances chemo-sensitivity in hepatocellular carcinoma. Cancer Biol Ther 2007, 6:1900-1907.

18. Yeo EJ, Ryu JH, Chun YS, Cho YS, Jang IJ, Cho H, Kim J, Kim MS, Park JW: YC-1 induces $S$ cell cycle arrest and apoptosis by activating checkpoint kinases. Cancer Res 2006, 66:6345-6352.

19. Wang SW, Pan SL, Guh JH, Chen HL, Huang DM, Chang YL, Kuo SC, Lee FY, Teng CM: YC-1 [3-(5'-Hydroxymethyl-2'-furyl)-1-benzyl Indazole] exhibits a novel antiproliferative effect and arrests the cell cycle in G0-G1 in human hepatocellular carcinoma cells. J Pharmacol Exp Ther 2005, 312:917-925.

20. Tung JN, Cheng YW, Hsu CH, Liu TZ, Hsieh PY, Ting LL, Ko HL, Chang YJ, Chiou JF, Wu AT: Normoxically overexpressed hypoxia inducible factor 1-alpha is involved in arsenic trioxide resistance acquisition in hepatocellular carcinoma. Ann Surg Oncol 2011, 18:1492-1500.

21. Subramaniam A, Shanmugam MK, Perumal E, Li F, Nachiyappan A, Dai X, Swamy SN, Ahn KS, Kumar AP, Tan BK, et al: Potential role of signal transducer and activator of transcription (STAT)3 signaling pathway in inflammation, survival, proliferation and invasion of hepatocellular carcinoma. Biochim Biophys Acta 1835, 2013:46-60.

22. Tai WT, Cheng AL, Shiau CW, Huang HP, Huang JW, Chen PJ, Chen KF: Signal transducer and activator of transcription 3 is a major kinase-independent target of sorafenib in hepatocellular carcinoma. J Hepatol 2011, 55:1041-1048.

23. Gu FM, Li QL, Gao Q, Jiang JH, Huang XY, Pan JF, Fan J, Zhou J: Sorafenib inhibits growth and metastasis of hepatocellular carcinoma by blocking STAT3. World J Gastroenterol 2011, 17:3922-3932.

24. Ezzoukhry Z, Louandre C, Trecherel E, Godin C, Chauffert B, Dupont S, Diouf M, Barbare JC, Maziere JC, Galmiche A: EGFR activation is a potential determinant of primary resistance of hepatocellular carcinoma cells to sorafenib. Int J Cancer 2012, 131:2961-2969.

25. Blivet-Van Eggelpoel MJ, Chettouh H, Fartoux L, Aoudjehane L, Barbu V, Rey C, Priam S, Housset C, Rosmorduc O, Desbois-Mouthon C: Epidermal growth factor receptor and HER-3 restrict cell response to sorafenib in hepatocellular carcinoma cells. J Hepatol 2012, 57:108-115.

26. Siegel AB, Olsen SK, Magun A, Brown RS Jr: Sorafenib: where do we go from here? Hepatology 2010, 52:360-369.

27. Li Y, Tang Y, Ye L, Liu B, Liu K, Chen J, Xue Q: Establishment of a hepatocellular carcinoma cell line with unique metastatic characteristics through in vivo selection and screening for metastasis-related genes through cDNA microarray. J Cancer Res Clin Oncol 2003, 129:43-51.

28. Chou TC, Talalay P: Quantitative analysis of dose-effect relationships: the combined effects of multiple drugs or enzyme inhibitors. Adv Enzyme Regul 1984, 22:27-55.

29. Kong J, Kong J, Pan B, Ke S, Dong S, Li X, Zhou A, Zheng L, Sun WB: Insufficient radiofrequency ablation promotes angiogenesis of residual hepatocellular carcinoma via HIF-1alpha/VEGFA. PLoS One 2012, 7:e37266.

30. Chen KF, Tai WT, Liu TH, Huang HP, Lin YC, Shiau CW, Li PK, Chen PJ, Cheng AL: Sorafenib overcomes TRAIL resistance of hepatocellular carcinoma cells through the inhibition of STAT3. Clin Cancer Res 2010, 16:5189-5199.

31. Wei G, Wang M, Hyslop T, Wang Z, Carr BI: Vitamin K enhancement of sorafenib-mediated HCC cell growth inhibition in vitro and in vivo. Int $J$ Cancer 2010, 127:2949-2958.

32. Hartmann JT, Haap M, Kopp HG, Lipp HP: Tyrosine kinase inhibitors - a review on pharmacology, metabolism and side effects. Curr Drug Metab 2009, 10:470-481.

33. Cervello M, Bachvarov D, Lampiasi N, Cusimano A, Azzolina A, McCubrey JA, Montalto G: Molecular mechanisms of sorafenib action in liver cancer cells. Cell Cycle 2012, 11:2843-2855.

34. To KF, Chan MW, Leung WK, Ng EK, Yu J, Bai AH, Lo AW, Chu SH, Tong JH, Lo KW, et al: Constitutional activation of IL-6-mediated JAK/STAT pathway through hypermethylation of SOCS-1 in human gastric cancer cell line. Br J Cancer 2004, 91:1335-1341.

35. Chen KF, Chen HL, Shiau CW, Liu CY, Chu PY, Tai WT, Ichikawa K, Chen PJ, Cheng AL: Sorafenib and its derivative SC-49 sensitize hepatocellular carcinoma cells to CS-1008, a humanized anti-TNFRSF10B (DR5) antibody. $\mathrm{Br}$ J Pharmacol 2013, 168:658-672.

36. Yang SF, Wang SN, Wu CF, Yeh YT, Chai CY, Chunag SC, Sheen MC, Lee KT: Altered p-STAT3 (tyr705) expression is associated with histological grading and intratumour microvessel density in hepatocellular carcinoma. J Clin Pathol 2007, 60:642-648.

37. Schneller D, Machat G, Sousek A, Proell V, van Zijl F, Zulehner G, Huber H, Mair M, Muellner MK, Nijman SM, et al: p19(ARF) /p14(ARF) controls oncogenic functions of signal transducer and activator of transcription 3 in hepatocellular carcinoma. Hepatology 2011, 54:164-172.

38. Strowski MZ, Cramer T, Schafer G, Juttner S, Walduck A, Schipani E, Kemmner W, Wessler S, Wunder C, Weber M, et al: Helicobacter pylori stimulates host vascular endothelial growth factor-A (vegf-A) gene expression via MEK/ERK-dependent activation of Sp1 and Sp3. FASEB J 2004, 18:218-220.

39. Liang Y, Zheng T, Song R, Wang J, Yin D, Wang L, Liu H, Tian L, Fang X, Meng $X$, et al: Hypoxia-mediated sorafenib resistance can be overcome by EF24 through Von Hippel-Lindau tumor suppressor-dependent HIF-1alpha inhibition in hepatocellular carcinoma. Hepatology 2013, 57:1847-1857.

doi:10.1186/1476-4598-13-7

Cite this article as: Kong et al:: YC-1 enhances the anti-tumor activity of sorafenib through inhibition of signal transducer and activator of transcription 3 (STAT3) in hepatocellular carcinoma. Molecular Cancer 2014 13:7.

\section{Submit your next manuscript to BioMed Central and take full advantage of:}

- Convenient online submission

- Thorough peer review

- No space constraints or color figure charges

- Immediate publication on acceptance

- Inclusion in PubMed, CAS, Scopus and Google Scholar

- Research which is freely available for redistribution 